\title{
MEASUREMENT AND ESTIMATION OF THE ANGLE OF ATTACK AND THE ANGLE OF SIDESLIP
}

\author{
Stanisław POPOWSKI, Witold DĄBROWSKI \\ Institute of Aviation, Al. Krakowska 110/114, 02-256 Warsaw, Poland \\ E-mails: witold.dabrowski@ilot.edu.pl; stanislaw.popowski@ilot.edu.pl (corresponding author)
}

Received 12 March 2014; accepted 20 January 2015

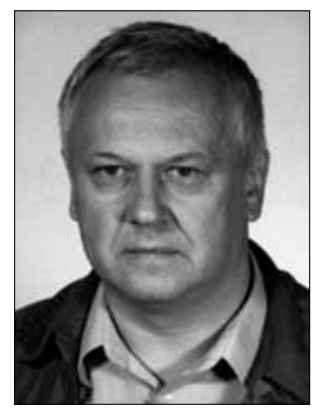

Stanislaw POPOWSKI, PhD Eng.

Education: a graduate of the Faculty of Mechanical Energy and Aviation at the Warsaw University of Technology in 1978.

Affiliations and functions: assistant professor at the Institute of Aviation, Warsaw. Research interests: avionic measuring systems, in particular measurement based on inertial technology.

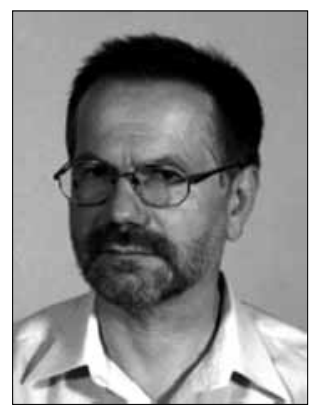

\section{Witold DĄBROWSKI, MSc Eng.}

Education: a graduate of the Faculty of Mechanical Energy and Aviation at the Warsaw University of technology in 1979.

Affiliations and functions: senior research technical specialist at the Institute of Aviation, Warsaw.

\begin{abstract}
The paper presents issues concerning the estimation of the angle of attack and the angle of sideslip on a flying object board. Angle of attack and sideslip estimation methods which are based on measurements of linear velocity components of an object with the Earth's coordinates and on attitude angles of the object are presented. Both of these measurements originate from the inertial navigation system, and velocity measurement is obtained from the satellite navigation system. The idea of applying inertial and satellite navigation for the estimation of attack and sideslip angles is presented. Practical comparison of these estimation methods has been conducted based on logged parameters of a flight onboard a Mewa aircraft. Development proposals for these methods are presented as well.
\end{abstract}

Keywords: estimate, angle, attack, sideslip, measurement.

\section{Introduction}

Angles of attack and sideslip are very important parameters determining the safety of the flight. Knowledge of them allows improving stability and controllability of the aircraft and it is especially important during take-off and landing. To avoid a situation leading to a critical angle of attack, modern aircraft are equipped with measurement and warning systems designed to alert the pilot or to automatically control the plane by limiting dangerous flight ranges. Zero sideslip angle assures a symmetrical aircraft airflow resulting in the least amount of drag. It is important for optimizing fuel consumption in longrange flights. In the case of a single static pressure port (located on one side of the hull) a sideslip angle of $1 \mathrm{deg}$ can cause a measurement error of barometric altitude of the order of $200 \mathrm{~m}$ (US 6561020 B2 2003).

The angle of attack $\alpha$ is the angle between the longitudinal axis $x$ and the projection of the vector speed $\overrightarrow{\bar{V}}$ on the plane of symmetry $x, z$ (Fig. 1 ). The angle is positive, when the vector component $\vec{V}$ along the normal axis $z$ is positive. The span of the attack angle is contained within the range $-\pi<\alpha \leq \pi$. According to 
(Polska... 1984), the angle of sideslip $\beta$ is the angle between the vector of speed relative to the air $\vec{V}$ and the plane of the aircraft, $x, z$. The angle is positive when the speed vector component $\vec{V}$ of transverse axis $y$ is positive. The sideslip angle span is included in the range:

$$
-\frac{\pi}{2} \leq \beta \leq \frac{\pi}{2}
$$

The disadvantages of the measurement of the sideslip angle applying classic methods using Pivoted Vane type sensor mounted on an external mounting are commonly known. One of the main defects is the measurement of the sideslip angle at the place of installation of the sensor, sometimes the angle measures this way significantly differs from actual values. In addition, the accuracy of a typical mechanical sensor is small. Besides, sometimes there is not enough room to fit a typical sensor (e.g. a small unmanned plane) or the actual sideslip angle measurement is conducted only for the duration of

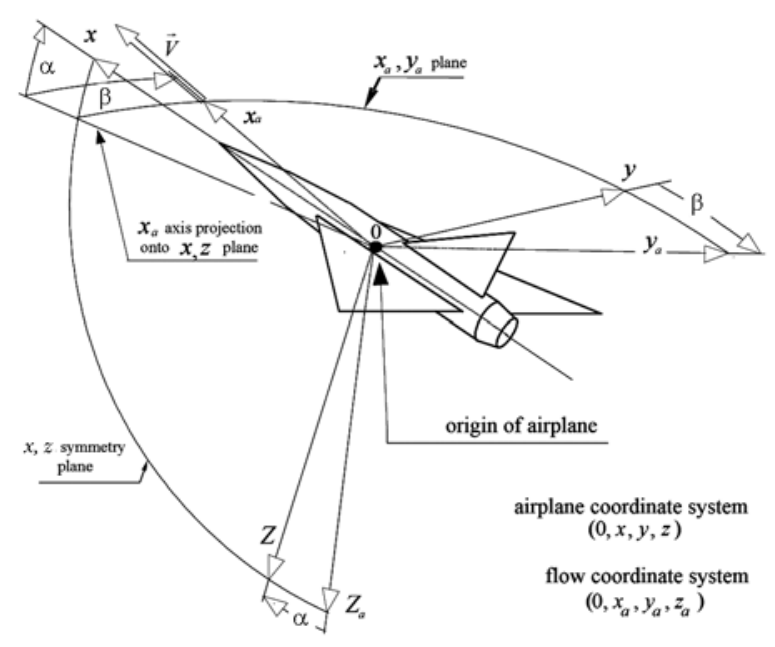

Fig. 1. Definition of angle of attack and angle of sideslip (Polska... 1984)

a)

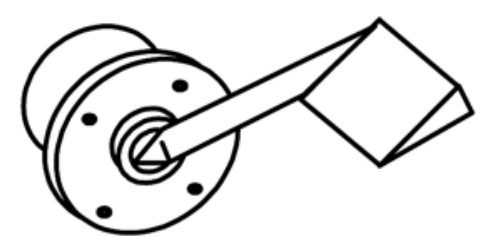

b)

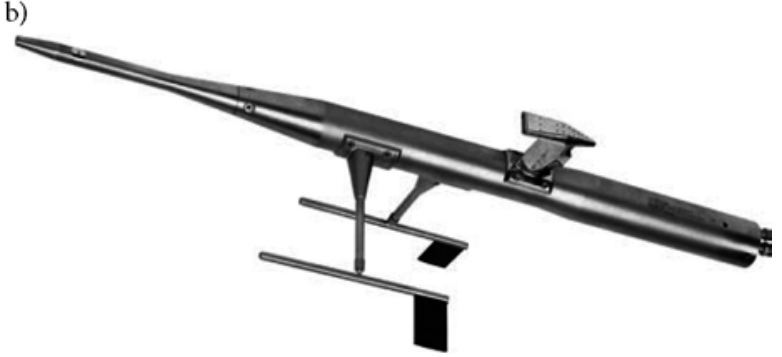

Fig. 2. Pivoted vanes: angle of attack (a) and angle of attack and sideslip angle (b) (Company... 2002) the fly test and installation of the sensor would be temporary. Below the various methods of measurement and estimation of the actual sideslip angle using appropriate signals received from the inertial navigation system and the satellite navigation system are presented. These methods, may prove to be useful in specific situations.

\section{Methods of measurement and estimation of angle of attack and sideslip angle}

For direct measurement of the angle of attack and sideslip mostly Pivoted Vanes or pressure type sensors are used (p. 2.1 and 2.2 below). Also servo sensors slottype that may be pressure-powered or electric can be applied. The sideslip angle can also be estimated on the basis of measurements of transverse acceleration, roll angle, yaw angular velocity and linear velocity (p. 2.4), and the angle of attack and sideslip angle can also be calculated from the measured or estimated components of linear velocity in the airplane's coordinate system (p. 2.5).

\subsection{The measurement of angles using sensor type pivoted vanes}

These sensors are placed either on the common boom with receivers of the total and static pressure, or can also be mounted in experimentally selected locations on the hull. The measurement principle is based on determining the angular displacement of metal, mass balanced swinging flap with a cuneiform profile, which sets itself parallel to incoming air, by means of a potentiometric transducer or Synchro (Fig. 2). The sensors of this type determine the angle of attack and the sideslip angle within the range of $\pm 30^{\circ}$ with an accuracy of $\pm 0.25^{\circ}$. They measure the angles correctly for flight speed from dozens to more than several hundred meters per second. Currently, pivoted vane type sensors are quite commonly used in spite of many serious disadvantages. One of them is that their output signal is disturbed by aerodynamic vibrations of a free flap. The maximum amplitude of these oscillations is about $0.3 \div 0.5^{\circ}$, and their frequency is within the limits of a few Hertz.

Another significant disadvantage of Pivoted Vane type sensors is the change of the dynamic characteristics depending on the speed and altitude of the flight.

\subsection{Measurement of angles by using a differential- pressure tube type sensor}

the second means for measuring angles is using a pneumometric sensor. The principle of operation of this sensor is based on measuring differential pressure (Fig. 3) on the flowed geometrical body. The axis of this sensor is parallel to the longitudinal axis of the aircraft. The front surface of the probe has the shape of a cone or a hemisphere (Fig. 3). It is mounted at the end of the Pitot tube and it takes in air pressure 
with two pairs of holes placed symmetrically in relation to the central hole (in vertical and horizontal planes with respect to the aircraft). The difference of pressure measured in the vertical plane is used to determine the angle of attack, and the difference of pressure measured in the horizontal plane - to measure the sideslip angle. Angles of attack and sideslip can be determined from (Dąbrowski, Popowski 2013):

$$
\alpha=\frac{p_{\alpha 1}-p_{\alpha 2}}{k_{1}\left(p_{3}-\frac{p_{\beta 1}+p_{\beta 2}}{2}\right)} ; \beta=\frac{p_{\beta 1}-p_{\beta 2}}{k_{2}\left(p_{3}-\frac{p_{\beta 1}+p_{\beta 2}}{2}\right)} .
$$

The $k_{1}$ and $k_{2}$ coefficients are chosen experimentally. In the case of using a hemisphere-shaped probe, the best results are obtained by the arrangement with the holes on the arc based on the angle of $90^{\circ}$, and in the case of the probe with a cone shape the best results are achieved by using the cone with the apex angle value of $90^{\circ}$.

\subsection{Measurement of angles by using a slot-type sensor (Null-seeking pressure sensor)}

in recent years, slot-type aerodynamic angle sensors have been extensively used. The main element of such sensors is a rotating, cylindrical probe with its interior divided into two chambers (Fig. 4). Each of the chambers is connected with the environment through a slot placed on the surface of the probe. Two sections of the slots are placed symmetrically along the probe. The probe axis must be perpendicular to the plane of the measurement of angle $\alpha$ or $\beta$, and the slots must face the flowing stream. The difference of pressure in the chambers will be present until a slots section is symmetric to the stream. Probe rotation is forced by torque proportional to the pressure difference in the chambers, or by using the electric servo, which turns the probe until it reaches its state of balance. The rotation angle of the probe is converted into an electric measuring signal. Slot sensors with a pneumatic drive are characterized by a simple design and good dynamic and accuracy characteristics. Their main disadvantage is the airflow through the interior of the probe, which may lead to dust and moisture settling inside the space probe.

Slot sensors with electric servo do not require an airflow. The probe is connected by channels to chambers of the electrical differential pressure transducer, which should have high stability and low threshold sensitivity.

\subsection{Determination of the sideslip angle through indirect measurement of: transverse acceleration $a_{y}$, roll $\Phi$, yaw angular speed $p$ and speed of flight $V$}

Figure 5 illustrates the operating accelerations in the direction of the $y$-axis of the aircraft during a typical flight.

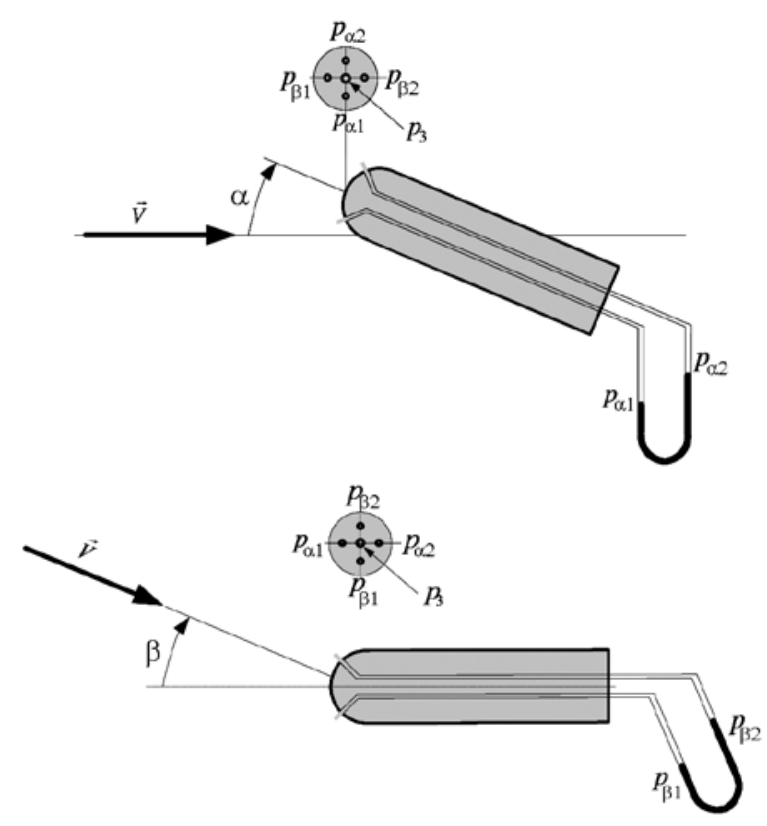

Fig. 3. Pressure method for the measurement of angles of attack and sideslip

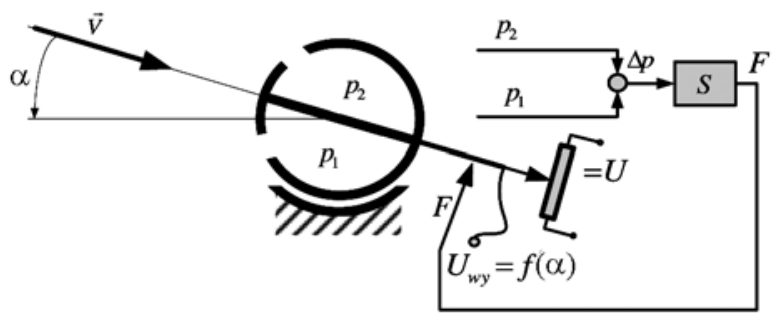

Fig. 4. Scheme of slot sensor of the angle of attack with pneumatic drive

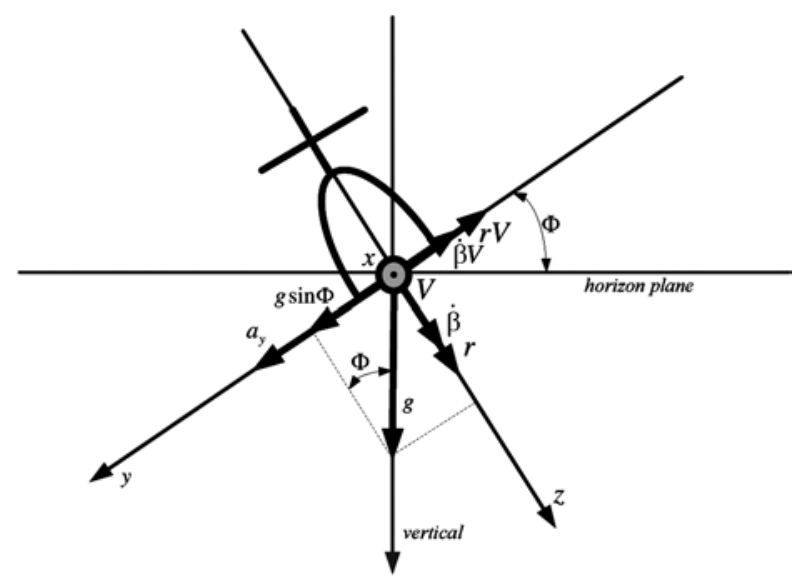

Fig. 5. Accelerations acting alongside with y axis of an aircraft 
The accelerometer measuring this axis will register acceleration, as the result of: Earth's gravity (gsin $\Phi)$, nonlinear movement $(r V)$ and a resultant factor of sideslip angle speed $(\dot{\beta} V)$.

In accordance with Figure 5, accelerations acting along the $y$ axis of the aircraft can be formulated as:

$$
a_{y}+g \sin \Phi-r V-\dot{\beta} V=0
$$

After conversion, the velocity of the sideslip angle can be estimated as:

$$
\begin{aligned}
& \dot{\beta} V=a_{y}+g \sin \Phi-r u ; \\
& \dot{\beta}=\frac{a_{y}}{V}+\frac{g \sin \Phi}{V}-r ; \\
& \dot{\beta}=\frac{a_{y}}{V}+\frac{g \Phi}{V}-r=\left(\frac{a_{y}+g \Phi}{V}\right)-r .
\end{aligned}
$$

Expression (3) can be used as a basis for building the sideslip angle estimation. A diagram of such a system is shown in Figure 6.

In expression (3) a simplification can be made. In flight situations, mostly coordinated with a temporary slip at times, the following relationship takes place:

$$
V r=g \Phi \text {. }
$$

Then expression (3) is simplified as:

$$
\dot{\beta}=\frac{a_{y}}{V} \text {. }
$$

The structure of the sideslip angle estimator in a simplified version is presented in Figure 7.

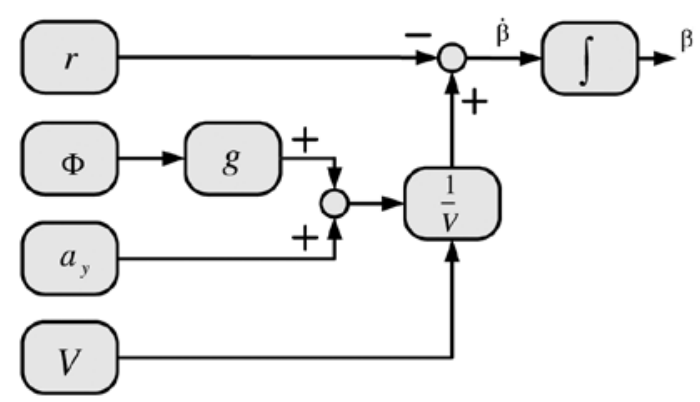

Fig. 6. Scheme of the sideslip angle estimation system (US 4094479 1978)

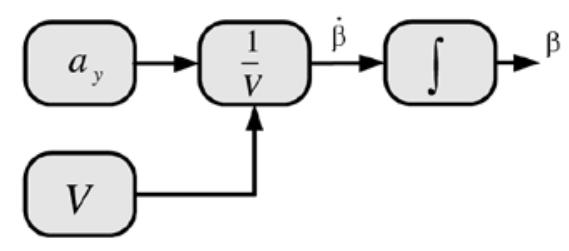

Fig. 7. Idea of a more simple system of sideslip angle estimation
2.5. Determination of the angle of attack and sideslip angle with linear speed measurement according to aircraft coordinates

Attack and sideslip angles can be determined from linear velocities of aircraft measured according to aircraft coordinates in accordance with the relationship (6) resulting from Figure 1:

$$
\alpha=\operatorname{arctg}\left(\frac{w}{u}\right) ; \beta=\operatorname{arctg}\left(\frac{v}{u}\right),
$$

where the values of $u, w$ speeds in the object's coordinates are designated with components of linear speeds measured in relation to the ground after the transformation of the airplane's coordinates using transformation matrix $E(7)$ :

$$
\left[\begin{array}{c}
u \\
v \\
w
\end{array}\right]=[E]\left[\begin{array}{c}
V_{N} \\
V_{E} \\
V_{Z}
\end{array}\right] .
$$

Knowledge of orientation angles is needed to calculate the transformation matrix (for example, from the AHRS). Speeds relative to the ground are measured by a GPS receiver. When all angles of orientation and components of speed in normal Earth's coordinates are known, nothing stands in the way estimating the components in the airplane coordinates:

$$
\begin{aligned}
u= & (\cos \Theta \cdot \cos \Psi) V_{N}+(\cos \Theta \cdot \sin \Psi) V_{E}-(\sin \Theta) V_{Z}, \\
v= & (-\cos \Phi \cdot \sin \Psi+\sin \Phi \cdot \sin \Theta \cdot \cos \Psi) V_{N}+ \\
& (\cos \Phi \cdot \cos \Psi+\sin \Phi \cdot \sin \Theta \cdot \sin \Psi) V_{E}+ \\
& (\sin \Phi \cdot \cos \Theta) V_{Z}, \\
w= & (\sin \Phi \cdot \sin \Psi+\cos \Phi \cdot \sin \Theta \cdot \cos \Psi) V_{N}+ \\
& (-\sin \Phi \cdot \cos \Psi+\cos \Phi \cdot \sin \Theta \cdot \sin \Psi) V_{E}+ \\
& (\cos \Phi \cdot \cos \Theta) V_{Z} .
\end{aligned}
$$

In this case, the process of determining the sideslip angle starts with the measurement of: $\Theta, \Phi, \Psi, V_{N}, V_{E}, V_{Z}$ and then using relations in (7) for the calculation of $u, v, w$ which leads directly to the desired value according to equations in (6).

As it is shown in the presented inertial method, the sideslip angle estimation problem is a problem of attitude angle measurement and of the estimation of linear speeds in the normal Earth's coordinate system or in the coordinates of the object. To measure the angles of orientation an inexpensive AHRS type (Attitude Heading Reference System) system can be used. These systems are relatively cheap because they are based on the micromechanical sensors of acceleration and angular velocity. These systems use magnetometers for yaw correction. Unfortunately, the structure of these devices is limited in relation to inertial navigation systems. They do not possess a navigation path, and, therefore, do not 


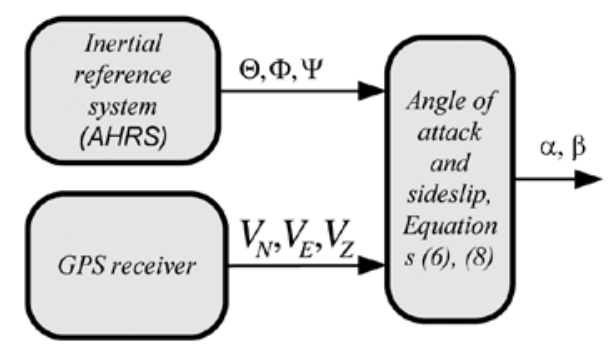

Fig. 8. Usage of a GPS receiver for the estimation of the angle of attack and sideslip angle

have the components of linear speeds available. In turn, a simple navigation system measures exactly three components of linear speed (i.e. message \$PGRMV NMEA in Garmin receivers). Therefore, in this configuration, the slip angle can also be estimated from relationships (6) and (8) (Fig. 8).

The disadvantage of this configuration can become evident for two reasons: a relatively slow delivery of components of linear speed from a GPS receiver (usually every 1s), and momentary interruptions of a GPS receiver, which can lead to disturbances in the slip angle estimation. In order to prevent these phenomena, we propose to extend the structure of Figure 8 by including the estimates of linear velocity, which also would use the information from static and dynamic pressure sensors and a GPS receiver. This structure is presented in Figure 9. Since the effects of the wind are omitted in the structure, the algorithms presented in (Popowski, Dąbrowski 2011) can be used to take them into account.

\section{Experimental verification of the selected methods of measurement and estimation of the attack and sideslip angles}

Data had been obtained during the tests carried out in Rzeszow, on Mewa board. Off-line mode calculations were carried out using a data set of registration obtained using measurements from: integrated attitude system IG-500N, attitude system VRU-BB-P, air data system CA05 and a GPS receiver. The results of the angle of attack and sideslip calculations are presented in Figure 10. Calculations take into account the estimated speeds of wind by modifying formula (7) in the following way:

$$
\left[\begin{array}{c}
u \\
v \\
w
\end{array}\right]=[E]\left[\begin{array}{c}
V_{N}-W \cos \Psi_{w} \\
V_{E}-W \sin \Psi_{w} \\
V_{Z}-W_{v}
\end{array}\right],
$$

where $W$ represents estimated wind speed on the horizontal plane; $\Psi_{w}$ - estimated wind direction, $W_{v}-$ estimated vertical wind.

\section{Conclusions}

The simplest and most effective methods are a direct measurement of the attack and sideslip angles by means of

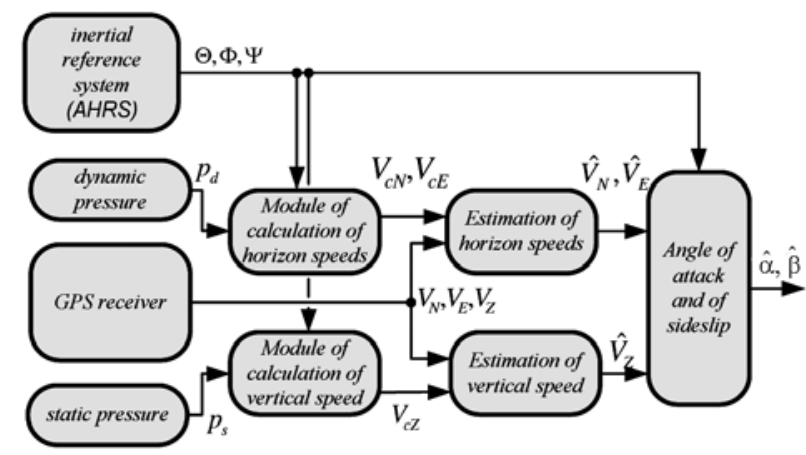

Fig. 9. System of estimation of the angle of attack and sideslip by using a GPS receiver, an inertial reference system and a sensor of static and dynamic pressure

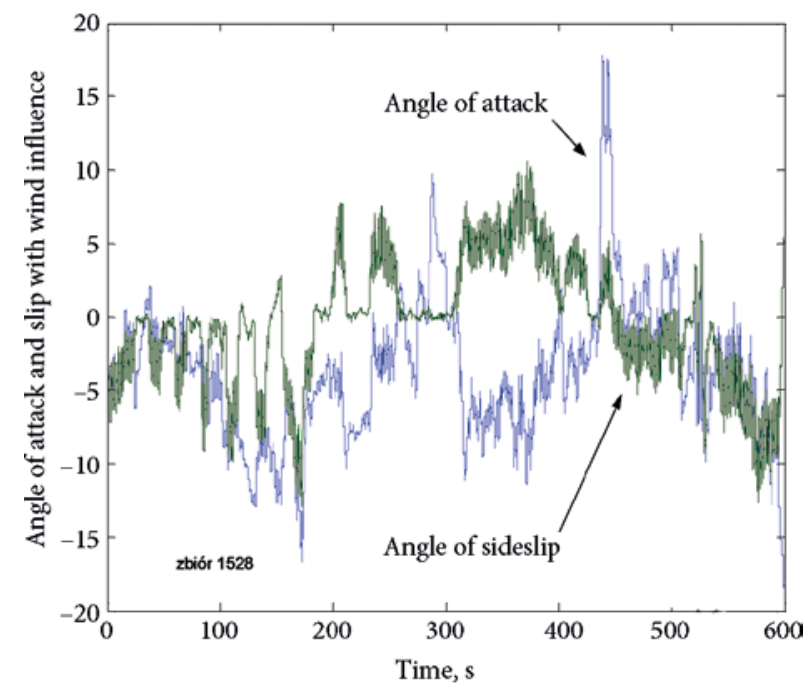

Fig. 10. Calculated angles of attack and sideslip with air speed taken into account

the Pivoted Vanes probe or pressure methods. If a direct measurement is impossible and the flight parameters are available, in accordance with equations (3), (6) and (8), by using the structures illustrated in Figures 6, 7, 8 and 9, it is possible to estimate the attack and sideslip angles. To know the actual attack and sideslip angles of the object, that would be independent of the mounting location of the probe, inertial measuring methods ought to be used.

They may be supplemented by linear speed measurement using a GPS receiver, as shown in Figure 8. Position (Pietrow et al. 1975) of the bibliography presents an even easier measurement system for the attack and sideslip angles, which only uses the measurements based on linear accelerometers. The simple methods described here may be particularly interesting when applied to small unmanned objects, where it is difficult to install external sensors (flap or pressure type). The essential element, which has not been widely discussed (equation (9)), is the effect of the wind which ought to be taken into account in the estimated values of the attack and sideslip angles. 


\section{References}

Company handouts of Goodrich: MODEL 0092BH OR 0092BJ AIR DATA FLIGHT TEST BOOM. 2002.

Dąbrowski, W.; Popowski, S. 2013. Estimation of wind parameters on flying object, Pomiary Automatyka Robotyka (2): 552-557.

Pietrow, B. N.; Studniew, R. W.; Krymow, A. B. 1975. Opriedielenie ugłow ataki i skołżenia po signałam akcelerometrow, ustanowlenych na bortu letatielnowo apparata, Izvestiya Vysshikh Uchebnykh Zavedeniy "Priborostroyeniye" 18(10).

Polska Norma PN-83/L-01010.01: Mechanika Lotu Samolotów i Szybowców, Terminologia, Układy Wspótrzędnych i Kąty. 1983.

Popowski, S.; Dąbrowski, W. 2011. Wykorzystanie nawigacji inercjalnej i satelitarnej do estymacji kąta natarcia, Pomiary Automatyka Robotyka (2): 774-782.

US 4094479. Sideslip Angle Command SCAS for Aircraft. 1978.

US 6561020 B2. Method to Sideslip Angle and Correct Static Pressure for Sideslip Effects Using Inertial Information. 2003. 